\section{Alcohol and cardiovascular disease}

Proceedings of an international symposium, Scheveningen, Netherlands, I - 2 October 199|

Editors: J. Veenstra \& D.G. van der Heij

TNO Nutrition and Food Research, Zeist, Netherlands

TNO Nucrition and Food Research

ISBN 90-220-1060-0

NUGI 735

Pudoc Wageningen 1992

\section{Alcohol and fibrinolysis}

C. Kluft ${ }^{1}$, A.F.H. Jie ${ }^{1}$, T. Kooistra ${ }^{1}$, P. de Knijff', E.J.C. de Geus ${ }^{2}$, G. Schaafsma ${ }^{3}$ and J. Veenstra ${ }^{3}$

1 Gaubius Laboratory, IVO-TNO, P.O. Box 430, 2300 AK Leiden, Netherlands;

2 Department of Psychophysiology, Vrije Universiteit, Amsterdam, Netherlands;

${ }^{3}$ TNO Nutrition and Food Research, Zeist, Netherlands

Abstract

Epidemiological studies have shown an inverse relation between moderate alcohol use and the incidence of coronary heart disease. Potential mechanisms explaining that relation may be found in the haemostatic processes. One possible candidate is the fibrinolytic system which, as the natural anticoagulant system in the body, may be reinforced by moderate alcohol consumption. Such an effect might protect against thrombo-embolic complications.

From epidemiological and experimental studies into the effects of alcohol consumption it can be concluded that the long-term effects of alcohol consumption on fibrinolytic variables are moderate. The short-term or acute effects, however, are marked and involve two- to ten-fold changes in blood levels of some components.

A recent study has shown that alcohol induces significant short-term increases in levels of the tissue-type plasminogen activator (t-PA). The data further support the notion that this increase is mainly effective with moderate alcohol consumption.

It is postulated that this increased fibrinolysis potential can contribute to a reduction of the risk for thrombo-embolic disease in two ways.

1) The increase of t-PA, which lasts for 10-15 hours, can counteract new thrombotic events and enhance the removal of old clots locally in the vessels where t-PA is released by the endothelium.

2) The t-PA increase reaches a peak.in systemic activity in the early morning following an evening of moderate alcohol consumption. This peak coincides with the period of highest risk for myocardial infarction. It can be postulated that in moderate alcohol consumers the morning peak in frequency of infarctions is blunted, thus possibly explaining the lower risk in this group.

\section{Abbreviations}

dDAVP, desamino-D-arginine vasopression; LP(a), lipoprotein (a); PA, plasminogen activator; PAI-1, plasminogen activator inhibitor-1; t-PA, tissue-type plasminogen activator; $\mathrm{u}-\mathrm{PA}$, urokinase-type plasminogen activator. 


\section{Introduction}

In epidemiological studies an inverse relationship between moderate alcohol use and the incidence of coronary heart diseases has been found (reviewed in $44,51,56$ ) which has been confirmed very recently (24). In view of the importance of thromboembolic mechanisms in coronary heart disease potential mechanisms explaining this relationship might be found in the haemostatic processes.

In epidemiological and experimental studies several effects of alcoholic beverages on the natural anticoagulant system of fibrinolysis have been reported, which will be summarized in this paper. These effects will be interpreted retrospectively on the basis of recent data and recent insights into the mechanisms of fibrinolysis. In addition, some unpublished data from recent studies will be included.

\section{Fibrinolysis}

As a natural consequence of coagulation and local fibrin formation, the fibrinolytic system becomes activated and eventually generates the proteolytic enzyme plasmin which degrades the fibrin matrix of a clot or thrombus (see Fig. 1) $(30,31)$. These processes usually occur locally at the site of injury, and the fibrinolytic process is, to a large extent, regulated by local molecular mechanisms involving binding to, and assembly on, the fibrin structure. Also, acute local supply of factors from platelets and the endothelium plays an important role, while other factors are supplied by the blood. As can be seen in Fig. 1, the local supply mentioned mainly involves tissuetype plasminogen activator (t-PA) and plasminogen activator inhibitor-1 (PAI-1).

\section{Baseline situation}

In epidemiological and long-term experimental studies, the condition in baseline blood samples (taken at rest, usually in the morning) has been recorded, reflecting the resting state or potential of the fibrinolytic system in the circulating blood. The fibrinolytic potential in such samples has frequently been evaluated by using a functional analysis of the overall potency of the blood system in clot lysis methods where the whole blood (or a fraction) is clotted and lysis is quantified. In such methods the changes observed can be due to changes in various factors of the whole fibrinolytic cascade (see Fig. 1). In more recent years specific functional and immunological methods for practically all fibrinolytic components have become available. The major cell types supplying these factors to blood are hepatocytes (e.g. factor XII system, plasminogen, $\alpha_{2}$-antiplasmin, fibrinogen, histidine-rich glycoprotein, possibly PAI-1), endothelium (t-PA and possibly PAI-1) and possibly smooth muscle cells (PAI-1).

In the study of baseline blood, important local supply mechanisms that only happen during coagulation activation are not evaluated. Such effects can be

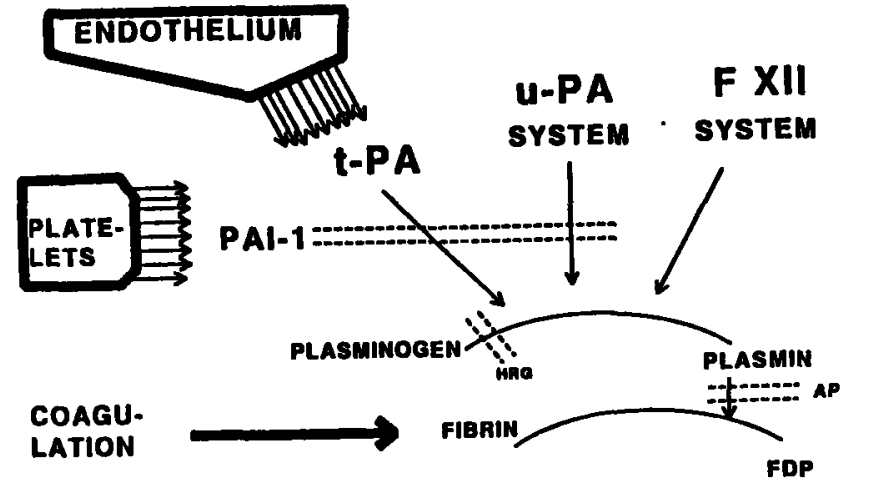

Fig. 1. Schematic representation of the fibrinolytic pathway. Fibrinolysis is the degradation of fibrin into soluble fibrin degradation products (FDPs). This degradation is achieved by plasmin formed from its precursor when needed by three different routes involving plasminogen activators. Abbreviations: uPA. urokinase-type plasminogen activator: t-PA. tissue-type plasminogen activator: F XII. factor XII: HRG. histidine-rich glycoprotein: AP. $a_{2}$-antiplasmin. The scheme indicates specifically the acute local supply of the factor t-PA from endothelium and the factor PAI-1 from platelets accumulating during coagulation. PAI-1 is an inhibitor capable of inhibiting both t-PA and u-PA.

evaluated, for instance, for t-PA to a certain extent by experimental stimulation through dDAVP infusion, exercise and venous occlusion.

In recent years, another approach has gained momentum and other methods have become available (32). This approach concerns the evaluation of specific degradation or activation products which mark the action or turn-over in the process. Such turnover happens continuously at a very low level and its condition and changes.can be recorded. Examples of products for evaluation are fibrin degradation products (FDPs) and plasmin- $\alpha_{2}$-antiplasmin complexes.

\section{Adaptation/acute effects}

Two important features of the fibrinolytic system which complicate evaluation are the occurrence of a sinusoidal diurnal fluctuation in appearance in blood of t-PA and PAI-1 and the possibility of rapid adaptation of synthesis and clearance of some of the fibrinolytic components.

For the circadian rhythm, analysis of a single blood sample should at least be standardized with respect to time of sampling. For factors with a circadian rhythm this carries the risk of missing changes (33). Only an evaluation of 2-3 samples sampled on well chosen times is adequate.

The rapid adaptation of the system should either be avoided or deliberately included in the approach, in a standardized way. Rather strict rules for sampling of blood have been formulated for the study of a baseline condition (34). Recent observations indicate that there can be residual effects of alcohol intake the evening before, which are especially evident in middle-aged volunteers. This has led to a more strict rule that alcohol consumption is to be avoided at least $24 \mathrm{~h}$ before 
sampling (34). It is possible that, depending upon instructions to participants, epidemiological studies are affected by carry-over effects of the participants' evenin habits, particularly with regard to alcohol consumption.

In evaluating fibrinolysis in relation to alcohol consumption we have strictly divided the studies into epidemiological and long-term experimental studies involvir analysis of 'base-line' blood samples on the one hand and a category of studies on acute effects on the other. A similar division has also proved to be valuable in the past for the effects of smoking: the acute and chronic effects on blood fibrinolytic activity were found to be opposite $(1,2)$.

\section{Recent improvements in laboratory methods}

It has only recently been fully appreciated how the coexistence in the circulating blood of the active enzyme t-PA and its active specific inhibitor PAl-1 (usually present in excess) influences existing pre-analytical technology and assay methods and what an ideal methodology should be.

It is theoretically obvious that the interaction of these components continues to manifest itself in vitro and reduces the recovery of active t-PA. To be able to identify the situation in the circulating blood, the situation needs to be 'frozen' immediately on sampling. The problem not only concerns specific assays for t-PA activity; because t-PA activity determines largely the spontaneous fibrinolytic activity of blood and blood fractions, it holds for all such methods. The necessity to stabilize t-PA can be calculated from kinetic data which have indicated that, in normal individuals, levels of both components are such that t-PA activity is halved (also in vitro) in 5-10 minutes. PAI-1 levels can be increased temporarily 10- to 1000-fold (circadian rhythm, trauma), and already with a 10-fold increase the time to reduce t-PA activity by $50 \%$ is ca. $30 \mathrm{~s}$ (31). Sampling at about $\mathrm{pH} 6.0$ is one way of stopping the interaction of t-PA and PAI-1, and the above calculation could be confirmed experimentally $(49,62)$. It is now clear that special blood sampling procedures are required for t-PA activity and blood fibrinolytic activity measurements and that any use of normal, usually frozen, plasma should be abandoned. In practice, preanalytical variation was controlled by rigorous standardization of blood handling and, in retrospect, the dilute blood clot lysis method requiring rapid handling and early dilution provided the best situation for maintenance of t-PA activity and for standardization of losses.

Another misfortune (retrospectively) concerned the fact that PAI-1 was recovered in euglobulin fractions (29). Earlier, it was generally believed that this fraction is poor in fibrinolysis inhibitors and hence a proper matrix for recovering t-PA and measuring its activity appropriately. We found that eventually only about $3 \%$ of the original t-PA activity was measured when euglobulin fractions were used. Accordingly, normal blood levels of t-PA activity currently reported are around 1 $\mathrm{IU} / \mathrm{ml}$, compared with previously reported normal values of ca. $0.05 \mathrm{IU} / \mathrm{ml}$.

The latest achievement in methodology concerns the inactivation of PAI-1 activity at a $\mathrm{pH}$ of around 3 and dilution of the sample which allows for appropriate recovery of plasma t-PA activity $(39,62)$. This new methodology in sampling and assay has been used only very recently in clinical studies and in an unpublished study which is reviewed below.

\section{Epidemiological studies}

Until recently, few epidemiological studies have included fibrinolytic variables in the laboratory test battery. It can be expected that ongoing studies will soon report further data, but at present only inconclusive data on fibrinogen concentrations and limited data on blood fibrinolytic activity and some specific plasma factors of fibrinolysis are available.

\section{Fibrinogen :}

After one report had suggested a relation between alcohol consumption and fibrinogen concentrations in blood, also a substantial number of reports showing no association appeared. Thus, in 1979, Meade et al. (41) reported a study among 160 men and 707 women aged 18-64 in North-West London in which a negative association between fibrinogen concentration and alcohol consumption was found. This association was confirmed in 1983 by Yarnell et al. (63) in a study among 670 men from the general population (aged 30-69) of Caerphilly, South Wales. However, the latter group found this association only when fibrinogen was

- Hetermined chemically, not with a clotting rate assay. The association was further detailed in 1988 by Rogers et al. (50) in a sub-sample of 665 men of the Caerphilly detailed in 1988 by Rogers et al. ( 50 ) in a data from a 7 -day weighed dietary inventory $(P<0.01)$ and with data from the complete cohort of 2512 completing a inventory $(P<0.01)$ and with data from the complete questionnaire $(P<0.001)$. A non-significant trend was reported in 1991 , while in 1983 Bain $(4)$, in a sub-set of 237 men in and 149 women of a studralia, and of a large London insurance firm found the Hospital, Brisbane, Australia, and of a large London insurance firm found the association only in women. No association between alcohol consumption and fibrinogen was observed in 1988 by Boniton-Kopp et al. (7) in 251 apparently healthy men aged 40-50 in Paris; in 1985 by Balleisen et al. (6) in 2880 men and 1306 women in the Münster Arteriosclerosis Study; in 1991 by Møller \& Kristensen 1306 women in the Munster Art l. (5) in 265-281 men (aged 45-64) drawn randomly from the practices of 16 general practitioners in the Speedwell study, with two methods of fibrinogen determination; in 1987 by Hamsten et al. (18) in 85 families with a proband with early myocardial infarction and 85 families randomly selected from the general Swedish population.

A variety of causes may underly these differences in observations: the effect is not large $(23,50,63)$ and can be obscured by various other aspects. Such aspects may be differences in accuracy of recording the alcohol consumption, the average level of 
alcohol consumption in a population, the type of beverage ( $80 \%$ beer in the study of Meade et al. (41)) and interaction of alcohol with other demographic and life-style characteristics such as smoking and dietary habits (6). The assay of fibrinogen may also be of significance as suggested by the discrepancy between methods in the Caerphilly study (63). Hamsten et al. (18) observed in the Swedish population that $51 \%$ of the variance of the plasma fibrinogen levels was accounted for by genetic heritability and concluded that there was a limited role for environmental influences.

\section{Plasminogen}

In a small cross-sectional study among 15 smokers and 15 non-smokers, all healthy males between 30 and 40 years of age, reported in detail previously (2), we observed in both groups a positive association between alcohol consumption and plasminogen concentration $(P<0.05$ and $P<0.02$, respectively) (Fig. 2). Alcohol consumption was generally moderate, not exceeding 1-2 consumptions a day on a regular basis, and weekend drinking.

In a later experimental study in 12 apparently healthy young men (21-29 years), we observed a small dose-dependent increase of 4 and $6.5 \%(P=0.01)$ in plasminogen concentration after consumption of 2 or 4 glasses of wine a day for 5 weeks (48). The values rose with increasing dose, but only the differences between abstaining and drinking were significant.

Recently, it was discovered that the LDL-like particle, lipoprotein (a), harbours an apoprotein with strong homology with plasminogen. Elevated plasma levels of this component had been identified as a risk marker for atherosclerotic coronary disease

Plasminogen (\%)

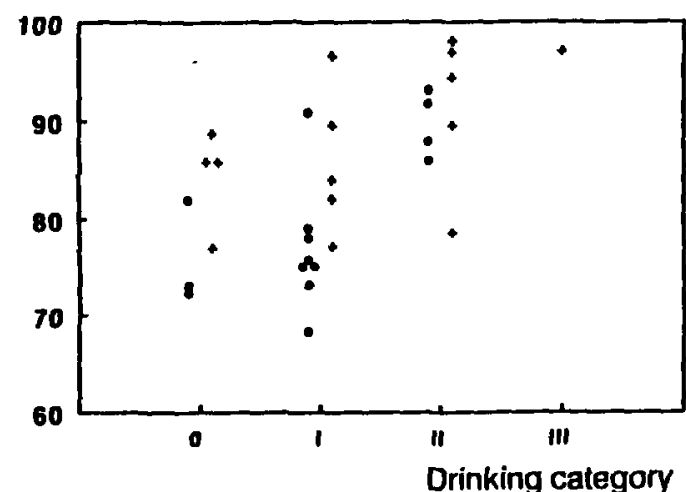

Fig. 2. Relation between plasma plasminogen concentration and drinking habits in healthy males aged 30 40. Plasminogen is expressed as percentage of a pooled plasma: drinking category: 0 , no alcohol: 1 . irregular small amount per week: II 1-3 drinks a day, weekend: III 1-2 drinks a day + weekends. non-smokers; + smokers. After: Allen et al. (2)
Table 1 Lipoprotein (a) concentrations in Inuit classified into categories with different level of habitual alcohol consumption. After: Johanson et al (25).

\begin{tabular}{lll}
\hline Alcohol category' & Number of individuals & LP(a), mean \pm SD (mg/dl) \\
\hline 1 & 15 & $8.7 \pm 11.1$ \\
2 & 85 & $9.2 \pm 10.4$ \\
3 & 33 & $8.0 \pm 6.5$ \\
\hline
\end{tabular}

1 , less than 1 consumption a day: $2,1-2$ consumptions: 3 , more than 2 consumptions a day: $P=$ 0.595 .

(54). A potential mechanism explaining the risk of high LP(a) might concern competition with plasminogen and inhibition of its action (37). In addition, it is possible that PAI-1 expression in endothelial cells is increased by LP(a) (14).

The observation that $L P(a)$ levels increased rapidly after ethanol withdrawal in alcoholics raises the possibility that ethanol intake is one of the few factors that affect the quite stable, genetically determined plasma LP(a) concentrations (27). Further investigations are required. In a recent study in 133 Inuit living in Greenland, no relation with habitual alcohol consumption was observed (see Table 1). Thus, in this population (25) there was no support for the above hypothesis.

\section{Blood fibrinolytic activity}

Until now only one report about blood fibrinolytic activity and alcohol consumption is known. Meade et al. (41) reported a positive association between fibrinolytic activity as measured by a dilute blood clot lysis technique and alcohol consumption. This association only concerned the 1601 men in the study and was not significant in the 707 women $(9,41)$, who had a lower average consumption (40).

These epidemiological data are hard to confirm because it is unlikely that further data on the dilute blood clot lysis time method will become available: this method is no longer in general use and is technically and logistically demanding, requiring fresh blood to be studied immediately. It can be expected that data on specific fibrinolysis factors possibly involved in the change of the blood fibrinolytic activity will become available. The dilute blood clot lysis method is sensitive to variation in various factors of fibrinolysis, including fibrinogen and plasminogen, but it is unlikely that the small differences in fibrinogen and plasminogen induced by alcohol (ca. $5 \%$ ) fully explain the observed changes reported by Meade et al. (41). Other factors with a large impact on the dilute blood clot lysis test such as, notably, a $a_{2}$-antiplasmin, plasminogen activator inhibitor-1 (PAI-1) and tissue-type plasminogen activator (t-PA) might be candidates.

Reported data indicate that $a_{2}$-antiplasmin is not likely to be involved. BonitonKopp et al. (7) noted a slight negative trend (not statistically significant), if anv effect at all, between alcohol consumption and $\alpha_{2}$-antiplasmin. Mettinger (42) found no 


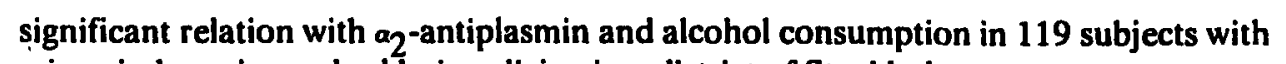
minor ischaemic cerebral lesions living in a district of Stockholm.

Data on PAI-1 are limited and provide at the very most a suggestion of alcohol dependence. Mettinger (42) found no relationship in the Stockholm population mentioned, and in a recent study among sedentary male workers recruited at the Free University of Amsterdam (aged 25-40) no correlation between PAI activity (after logarithmic transformation) and alcohol consumption was found $(r=0.0756$, $n=57, P=0.576$ ). The average alcohol consumption was $1.43 \pm 1.48$ (SD) glasses per day. The consumption recorded by questionnaire correlated with the consumption recorded on two occasions by a three-day diet diary method $(r=$ $0.509-0.767, P<0.0001)$. The relationship between alcohol consumption and PAI activity is shown in Fig. 3.

A trend becomes apparent only when regular drinkers (more than one glass a week) are studied separately $(r=0.2615, n=47, P=0.076)$, while comparison with the non-drinkers (below one glass/week) shows significance $(P=0.038)$. This suggestion of a relation is possibly supported by data from Tran-Thang et al. (53) who found elevated PAI-1 and normal t-PA values in alcoholics without signs of liver damage.

It can be concluded that the effects of alcohol on PAI-1 are not likely to contribute to an increased blood fibrinolytic activity, but may at the most exert an opposite trend in inhibiting lysis.

One of the remaining candidates is t-PA, which is expressed relatively well in the dilute blood clot lysis time assay. There is an acute increase in t-PA antigen upon alcohol consumption (see below), which effect might stabilize with regular alcohol

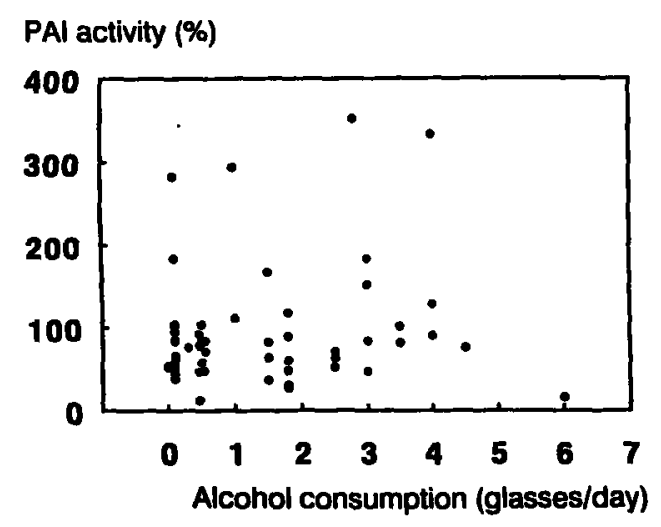

Fig. 3. Relation between alcohol consumption and PAI activity. PAI activity was recorded in morning blood samples $(09.00-11.00)$ and expressed as a percentage relative to a pooled plasma standard (290385) determined to neutralize $7.6 \mathrm{IU} / \mathrm{ml}$ of t-PA activity (60). Alcohol consumption is expressed as the average number of glasses of alcoholic beverages per day over the past three months. Subjects were recruited from the pool of male workers at the Free University of Amsterdam aged 25-40 and had not been involved in sport or fitness training during the preceding half year (17). consumption. With the new method for t-PA activity assessment mentioned above its possible chronic elevation can be verified in the future.

\section{Experimental studies}

\section{Long-term effects}

In a large, randomized, controlled trial, Burr et al. (8) studied in a single cross-over design the effects of 4 weeks of alcohol consumption as compared to 4 weeks of total abstinence in 48 men and 52 women aged 19-60. Alcohol consumption was not standardized and varied from lèss than two drinks a week to more than 20 per week (mean intake $18.4 \mathrm{~g}$ alcohol per day). The type of alcoholic beverage was left to choice. At the end of each experimental period a fasting blood sample was taken. No significant effect of alcohol consumption on fibrinogen was observed.

Pikaar et al. (48) confirmed the absence of an effect on fibrinogen in a study comprising four different standardized amounts of red wine during a five-week experimental period in 12 male volunteers between 21 and 29 years of age. The treatments consisted of 0,2 and 4 glasses of red wine per day. The effect of 'binge drinking was also studied over a period of five weeks in which 14 glasses of red wine were consumed each weekend. Plasminogen levels were found to increase moderately; only the differences (4-6.5\%) between abstinence and drinking were significant. t-PA activity in euglobulin fractions of plasma showed a large and dosedependent decrease. Additional analysis revealed an increase in PAI-1 activity (35). which might cause the reduction of euglobulin t-PA activity in view of the large impact of PAI-1 on evaluation of this variable with previous methods (see above).

It appears that a long-term increase in PAI-1 requires alcohol to be consumed over a prolonged period. Thus, a single episode of four days of wine consumption (58) was insufficient to cause an effect. Another question is how regular alcohol consumption needs to be to bring about an effect. Apparently, binge drinking on three days a week was insufficient to cause an effect that lasted until the time of sampling on the next Wednesday/Thursday (48). It would be interesting to see what degree of irregularity (abstinence for one day a week, two days a week, ...?) would prevent a long-term effect on PAI-1 in case of moderate alcohol consumption.

\section{Acute effects: A very acule effect by non-alcoholic factors?}

In 1960, Fearnley et al. (16) observed by chance an unusual prolonged blood clot lysis time after consumption of one glass of beer. Subsequently, an acute effect was delineated with a peak effect 2-3 hours after the consumption of beer, wine or cider confirmed by others $(3,45)$. Notably, the (very acute) effect was not present after consumption of gin, whisky or pure alcohol, which has been confirmed by others ( 3 , $12,16,45$ ). These data suggested that a non-alcoholic component is responsible. Consequently, Nilsson et al. (45) and, later, Anderson et al. (3) observed in vitro a 
Table 2. Inhibition of plasmin activity on fibrin plates (unpublished data).

\begin{tabular}{lll}
\hline Addition to plasmin & \multicolumn{2}{l}{ Lysed zone of fibrin plate after $18 \mathrm{~h}(\mathrm{~mm})$} \\
\cline { 2 - 3 } & in saline & in gelatin buffer \\
\hline $1: 1$ with saline & 14.2 & 15.2 \\
l:1 with $12 \%$ ethanol & 14.2 & 15.7 \\
1:1 with wine & 0 & 0 \\
1:20 with wine & 9.5 & 14.2 \\
\hline
\end{tabular}

Plasmin (purchased from KabiVitrum AB, Stockholm) was diluted to a concentration of $0.4 \mathrm{CU} / \mathrm{ml}$ in either $0.15 \mathrm{M} \mathrm{NaCl}$ or gelatin buffer $(0.05 \mathrm{M}$ sodium diethyl barbiturate. $0.10 \mathrm{M} \mathrm{NaCl}, 0.25 \%(w / v)$ gelatin (microbiological grade. Merck), $2.7 \mathrm{mM}$ EDTA adjusted to $\mathrm{pH} 7.8$ with $\mathrm{HCl}$ ). The solution was mixed $1: 1$ with either saline. $12 \%$ ethanol in saline. wine dialysed against saline pH 7.35, or wine diluted in saline. Pre-incubation: $15 \mathrm{~min}$ at $37^{\circ} \mathrm{C} .30 \mu l$ drops were spotted in triplicate onto bovine plasminogen-free fibrin plates (20) (093; Organon Teknika. Boxtel, Netherlands) and lysed zones

were measured after $18 \mathrm{~h}$ incubation at $37^{\circ} \mathrm{C}$

fibrinolysis-inhibiting effect of the beverages mentioned and identified a pectin substance (45) and polyphenols (46) as inhibitory substances whereas ethanol was found not to be inhibitory.

In vitro studies showed inhibition of the polyphenols (46) and wine components to be directed at plasmin (see Table 2) and to be more pronounced for t-PA-induced plasmin formation than for $\mathrm{u}-\mathrm{PA}$-induced plasmin formation $(45,46)$.

If we compare the inhibition of the non-alcoholic factors in vitro with the observed inhibition in vivo, it appears, however unlikely, that the pectin and polyphenol components penetrate in sufficient amounts into the blood to exert as strong an effect as observed after drinking the beverages. For example, Nilsson et al. (45) observed an average decrease in activity on fibrin plates from $7.5 \mathrm{~mm}$ to $3.0 \mathrm{~mm}$ (average effects presented in Table 1 of ref. 45) which is stronger than our inhibitory effect in vitro with $5 \%$ wine in a protein-rich milieu (gelatin buffer). Effects in vitro on purified enzymes reported by Nilsson are stronger but might be related to a lower content of other proteins in the in vitro milieu. Furthermore, Anderson et al. (3) noted in in vitro clot lysis experiments that there was hardly any further effect at dilution below $1 \%$ cider. The $60-80 \%$ inhibition by the strongest polyphenols is found around $5 \mu \mathrm{g} / \mathrm{ml}(46)$ which requires for Bulmer's 'Woodpeckers' cider, with a content of procyanidin polymers of $64 \mu \mathrm{g} / \mathrm{ml}$, an unrealistically high absorption to obtain the reported $60-80 \%$ inhibition with consumption of $750 \mathrm{ml}$ after 2 hours in blood (3).

The delineation of a very acute effect was in fact only based on studies of the time profile in a small number of individuals by Fearnley et al. (16) and Nilsson et al. (45). In retrospect, it can be concluded that a more prolonged effect was over-looked. This was because these researchers did not include a control group which is essential in view of the diurnal variation in blood fibrinolysis discovered in roughly the same time

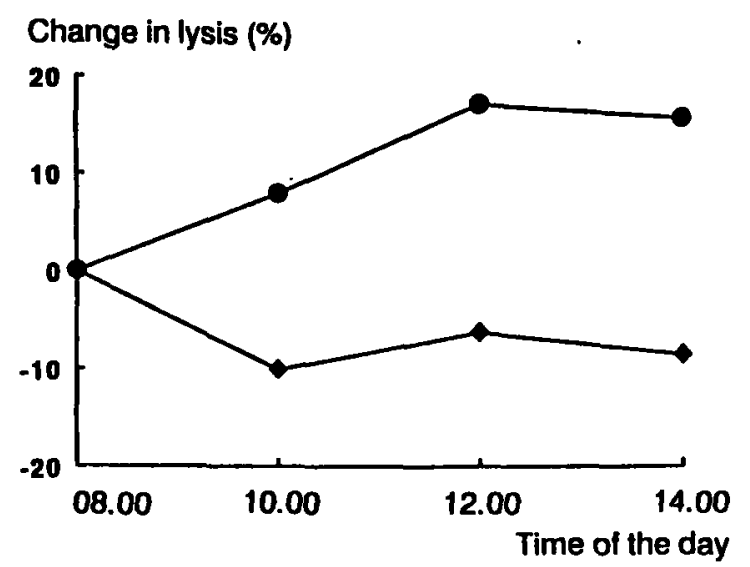

Fig. 4. Relationship between acute fibrinolytic activity changes and alcohol consumption. The percentage change from the starting point at 08.00 was calculated for 10 volunteers in two groups. The average of each group derived from Fig. 1 in Walter et al.'s paper is plotted. Dots: group with breakfast at 07.00 . low-fat dinner at 11.30 . total energy intakely $3.4 \mathrm{MJ}$ : diamonds: group with same breakfast and dinner and $1000 \mathrm{ml}$ beer between 07.45 and 08.15 . After: Walter et al. (61).

period (15). They considered the effect to be ended when the fibrinolytic activity 'returned to pre-treatment levels' not taking into account the diurnal increase that can be expected.

The effect of diurnal variation was already properly considered in 1979 by Walter et al. (61). The effect of beer on fibrinolytic activity clearly follows a slower pattern (Fig. 4. adapted from Walter's study); the decrease in fibrinolytic activity (when compared with a control session with a clear diurnal change) clearly extends beyond the 2-3 hour period.

On the other hand, the fact that the effect of ethanol per se on blood fibrinolytic activity was slower misled the investigators and was initially also overlooked. Hillbom et al. $(21,22)$ first reported in 1982 a decrease of euglobulin lysis on fibrin plates upon consumption of $1.5 \mathrm{~g} / \mathrm{kg}$ ethanol in fruit juice. The effect, documented for 12 healthy male volunteers aged 22-30, was apparent after 3 hours and was evaluated using data from a control session. In 1987, Olsen \& Østerud (47) confirmed and established the effect, further showing a dose-dependent effect on whole-blood clot lysis time. Ethanol in fruit juice was consumed over a prolonged period of 3 hours at rather high dosages, reaching a level of ca. 10 and $22 \mathrm{mM}$ in blood. Four hours after the start of drinking, clot lysis was already significantly retarded and remained so for the highest dose after 14 hours, whereas a control session showed no effects.

In a recent series of studies jointly performed by TNO Nutrition and Food Research and the Gaubius Laboratory, IVVO-TNO, we have identified the involvement of PAI-1 in the reduction of blood fibrinolytic activity and also showed 
that t-PA antigen levels could increase. Both effects could be obtained with gin as well as with wine and beer. The effects appeared to be more pronounced with older volunteers. We also observed that beer and wine gave a more rapid onset of effects than gin, which might further add to the understanding of the historical development of studies on the time profile of acute effects summarized above.

In one of the studies (57) we confirmed rapid effects of a combination of red port and wine. Two age groups of volunteers (20-30 and 45-55 years of age), each consisting of eight healthy men, were studied. All subjects received an aperitif (red port, $10 \mathrm{~g}$ of alcohol) half an hour before dinner and two glasses of red wine (20 gram of alcohol in total) during dinner. The results were compared with a similar session with the same volumes of mineral water on another day. These moderate amounts of alcohol resulted in a strong decrease in euglobulin t-PA activity (results dominated by PAI-1; see section Fibrinolysis) in the middle-aged group ( $-70 \%$, $P<0.001)$ and in a smaller decrease in the young men $(-29 \%, P=0.037)$ one hour after dinner. PAI activity levels increased significantly in the middle-aged men $(+60 \%, P=0.010)$, whereas the increase in young men $(+34 \%)$ was not significant $(P=0.144)$.

In a subsequent study (59), we confirmed the more extended time profile and observed that a period of one hour is too short for full effects on euglobulin t-PA activity, PAI activity and PAI-1 antigen. Six hours after a dinner with 2 glasses of red wine, a strong effect was seen with a $95 \%(P<0.001)$ reduction in t-PA activity, and a $230 \%(P<0.001)$ increase of PAI activity. We also observed an increase in t-PA . antigen, but no effects on variables of u-PA were noted (59), although an effect on uPA with other beverages might exist (52). It should be noted that in the above study the volunteers also consumed, one hour before the time point mentioned, two glasses of Hollands gin in combination with a snack, which complicates the interpretation of the effects and time profile.

Therefore, in a very recent study we analysed in eight healthy middle-aged men (aged 45-55) with multiple time points $(1,3,5,9,13$ hours) after $40 \mathrm{~g}$ alcohol contained in various alcoholic beverages taken during the evening meal the time profile of PAI activity, t-PA antigen and PAI-1 antigen. We observed a peak in the increases at 5 hours in all variables relative to the control experiment with mineral water. We used in the study $1000 \mathrm{ml}$ beer, $400 \mathrm{ml}$ red wine or $144 \mathrm{ml}$ Hollands gin and observed the increases for all drinks (unpublished). In a parallel study (unpublished) in collaboration with the Centre for. Human Drug Research (Leiden University) we also observed, during continuous infusion of ethanol in healthy young volunteers, an increase in PAI activity peaking after 5 hours.

There was a difference in the speed at which the PAI-1 and t-PA peak returned to normal. PAI-1 showed a much faster decline and t-PA antigen was still elevated the next morning. This was further substantiated with an assay of t-PA activity in acidified blood (62) which showed a significant increase in the morning in t-PA activity compared with the control session with mineral water.

\section{Mechanisms}

The fibrinolytic factors involved in effects of alcohol and discussed above are produced in the body by several cell types. They include endothelial cells ( $t-P A$ and PAI-1), hepatocytes (fibrinogen, plasminogen, PAI-1) and possibly smooth muscle cells (PAI-1).

\section{Direct effects}

Recently, Klöcking et al. (28) reported a direct effect of ethanol on t-PA release in the isolated perfused pig ear. The effect was significant starting at rather high dosages of $50 \mathrm{mM}$ ethanol. Such effects on the well-known very acute release of t-PA (time scale of minutes) differ among species (13): in our experiments t-PA antigen only increased on a time scale of hours. It seems unlikely, therefore, that this very acute t-PA release mechanism contributes directly to the observed increases in t-PA antigen.

Information on the direct effects of ethanol on the cells mentioned in culture is scanty. Laug (38) reported the effects of ethanol on the production of PA activity by bovine endothelial cells cultured in serum-free medium. After $18 \mathrm{~h}$ incubation alcohol increased PA in medium and cell lysates of endothelial cells from various origins. The effect was dose-dependent and started to be significant at higher levels of $0.2 \%(\mathrm{v} / \mathrm{v})$ ethanol and to be robust at $0.5 \%(\mathrm{v} / \mathrm{v})$. The type of PA was not identified and might be, in this case of cultured bovine endothelial cells, u-PA.

Some preliminary data on human endothelial cells and the human hepatoma cell

Table 3. Effects of ethanol on cell cultures.

\begin{tabular}{llrl}
\hline Cell type & Component & \multicolumn{2}{c}{ Level in conditioned medium with $0.1 \%(\mathrm{v} / \mathrm{v})$} \\
& & ethanol $(\%$ of control without alcohol) \\
\hline HUVEC & t-PA antigen & $24 \mathrm{~h}$ culture: & $103 \pm 11 \%(n=11)$ \\
HUVEC & PAl activity & $6 \mathrm{~h}$ culture: & $96 \pm 14 \%(n=11)$ \\
& $24 \mathrm{~h}$ culture: & $105 \pm 11 \%(n=11)$ \\
Hep G2 & PAl activity & $6 \mathrm{~h}$ culture: & $101 \pm 9 \%(n=7)$ \\
& & $24 \mathrm{~h}$ culture: & $99 \pm 16 \%(n=7)$ \\
\hline
\end{tabular}

Confluent HUVEC (human umbilical venous endothelial cells) cultures were used at second or third passage, and re-fed the day before the experiment with M199 medium, supplemented with 10\% human serum. $20 \mathrm{mM}$ HEPES. $100 \mathrm{IU} / \mathrm{ml}$ penicillin and $100 \mu \mathrm{g} / \mathrm{ml}$ streptomycin. Confluent Hep G2 cells as for HUVEC, with DMEM, supplemented with 10\% heat-inactivated foetal bovine serum. 100 $\mathrm{IU} / \mathrm{ml}$ penicillin and $100 \mu \mathrm{g} / \mathrm{ml}$ streptomycin. Conditioned medium was obtained by incubating cells in $5 \mathrm{~cm}^{2}$ dishes with $1 \mathrm{ml}$ of incubation medium either or not containing ethanol. t-PA antigen was measured using the Imulyse TMS I-PA kit from Biopool AB. Umeá. Sweden: PAI activity was assayed with the method of Verheijen et al. (60). 
line Hep G2, with low ethanol levels and an assay of the specific components t-PA and PAI-1, are summarized in Table 3. No effects on t-PA and PAI release were apparent. Similarly, we did not observe effects on production of plasminogen and fibrinogen by Hep G2 cells with low levels of ethanol $(0.07 \% \mathrm{v} / \mathrm{v})$. Such studies should be carried out more systematically and also include higher ethanol concentrations which in other studies have clearly shown to affect cellular metabolism (11).

Attention has also been given to the metabolites of ethanol, acetaldehyde and acetate. For human endothelial celis (HUVEC) we observed that acetaldehyde is toxic to cells at concentrations as low as $0.01 \%$ (cell death after 6 hours). This compares well to the experience with bovine endothelial cells reported by Laug (38) indicating that acetaldehyde is rather toxic to endothelial cells. For Hep G2 toxicity is apparent at $0.1 \%$ acetaldehyde or above after 24 hours. At low concentrations of acetaldehyde no effect on bovine endothelial cells has been found (38). For acetate, some effect on t-PA and PAI-activity in the medium of HUVEC has been reported at the relatively high concentration of $5 \mathrm{mM}$. At 8 hours PAI-1 activity had increased to $117 \%$ and at 24 hours to $127 \%$ of the controls; t-PA antigen at 24 hours had increased to $123 \%(36)$.

\section{Indirect effects}

For the induction of t-PA and PAI-1 synthesis numerous substances are known. Ethanol might be involved in generating one of these factors. No data are available to identify any intermediate factor for the induction of t-PA and PAI-1 synthesis. It can be mentioned that various effects of alcohol have been reported that might be considered to have a role. They include the generation of kinin (19), of endothelin-1 (26), and effects on growth hormone release $(10,55)$.

\section{Concluding remarks and perspectives}

Long-term effects of alcohol consumption on fibrinolytic variables, as observed in epidemiological and experimental studies, are of minor magnitude, especially when compared with robust effects observed acutely (peak at 5 hours) after alcohol consumption. Long-term effects concern factors produced by hepatocytes such as fibrinogen (inconsistent decrease) and plasminogen (slight increase). The impact of the small changes in blood levels of these factors for fibrinolysis is considered to be very moderate.

Long-term effects are documented for PAI-1 (its source can be multiple, including endothelium, but cannot be deduced from blood measurements), but not yet in full detail for endothelial t-PA, notably for its free fraction. For PAI-1 the regularity of alcohol consumption seems to be relevant for a stable long-term effect of increase. Recognition of this aspect indicates that for an adequate description of effects moderate alcohol cons.Imption should be defined more exactly with regard to regularity of daily consumption rather than in terms of average consumption.

Only one epidemiological study has documented increased blood fibrinolytic activity in moderate alcohol consumers. It is not clear yet how this relates to observations of increased PAI-1 in long-term experimental studies. It could be due to a simultaneous increase in t-PA and, notably, free t-PA (not yet studied by a specific assay). It should also be noted that it remains possible that in epidemiological studies the rather long-lasting acute effects contribute to the situation in the blood sample studied because of the alcohol consumed by the participants in the late afternoon or early evening. We recorded in this respect a late effect of increased t-PA activity, after alcohol consumption 'the early evening before', expressed in the morning after the increase of PAI-1 had ceased.

Acute effects involve both t-PA and PAI-1, with a robust peak in blood levels approximately 5 hours after alcohol consumption. The time profile, especially a rapid onset of reduced fibrinolytic activity with wine and beer compared with gin and ethanol, suggests non-alcoholic factors to be involved in the stimulation of PAI-1 appearance in blood. The direct inhibitory effect of non-alcoholic factors on fibrinolytic activity is not likely to be significant. The induction of inhibitory activity (PAI-1) seems to be enhanced at higher age and to be dose-dependent.

The data on t-PA and PAI-1 responses suggest that both factors differ in timing of effect. With moderate consumption the increase of PAI-1 induced by early-evening consumption has ceased the next morning, while heavier drinking still shows persistence of inhibition. The t-PA response is characterized by a slower reverting pattern and can, in the case of moderate drinking, result in significantly elevated t-PA activity in the early morning. This suggests that an early-morning t-PA increase counteracts the early-morning risk of acute myocardial infarction (and also stroke) and may be the basis for the observed lower risk for myocardial infarction among moderate alcohol users.

The increase in t-PA during the high PAI-1 peak in the earlier phase after drinking might also exert beneficial effects locally in the vascular tree. The local versus systemic situation is represented schematically in Fig. 5 . The locally produced

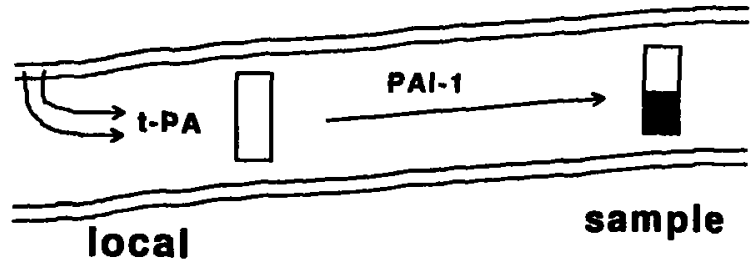

Fig. 5. Cartoon of a blood vessel indicating differences in the status of locally released t.PA Fig. 5. Cartoon of a blood vessel indicating differences in the status of locally released t-PA (free/active) and t-PA recovered in a sample obtained by venapuncture. The coexistence of t-PA PAI-1 results in inactivation of $50 \%$ of the t-PA (dark part of the bar) in case
about 360 seconds and with 10 -fold elevated PAl-1 in about 36 s. (See text.) 
PA is active and reacts only later with PAI-1. At the site of release, the alcohol thus creates an increased t-PA activity. Only gradually the PAI-1 diminishes t-PA activity, the more rapidly when PAI-1 is elevated as in the case of alcohol consumption. At the site of sampling in a larger vein the majority of t-PA derives from the circulation and a minor part is contributed locally by the endothelium in this vessel (an unfavourable wall/fluid ratio). When we sample arbitrarily at an average time of 5 min after release, t-PA activity is - normally, but especially with increased PAI-1 strongly reduced in this sampled blood. Comparing the normal situation with the situation after alcohol intake, when PAI-1 has increased 5-10-fold and t-PA 2-fold, it can be seen from Fig. 5 that the assessment of the situation in the sampled blood is not representative of the local situation. Locally, increased t-PA activity can be a dominating feature of alcohol responses.

These considerations of the results in view of the fibrinolytic mechanisms result in two postulates with regard to mechanisms that might contribute to a reduced risk of myocardial infarction upon moderate alcohol use.

1. Local t-PA increase. The every-day locally increased t-PA upon daily moderate alcohol consumption might contribute to the prevention of formation or to the dissolution of mural and other fibrin deposits in patients at risk, such as with unstable angina pectoris. The t-PA release might also be beneficial at more distant locations when PAI-1 is not greatly elevated, allowing t-PA to disseminate. This latter situation might exist when drinking is moderate. For experimental verification of the effectiveness of the mechanism in patients with increased fibrin deposits, such as patients with unstable angina pectoris. it may be possible to measure effects brought about by a single drinking session by $24-\mathrm{h}$ monitoring of fibrin degradation products compared to a control session.

2. Early-moming increase in systemic $t-P A$ activity. The increase in early-morning t-PA activity in the whole circulation might contribute specifically to a lowered risk of myocardial infarction. It may be possible to verify this by registering the 24 hour frequencies of myocardial infarction (and also stroke) in patients in relation to alcohol consumption. It is postulated that the early-morning peak in frequency of myocardial infarction is blunted in moderate alcohol consumers.

\section{Acknowledgements}

We are grateful to L.G. Johansen and M.P.M. de Maat for making available unpublished data. $\because$

\section{References}

1. Alten RA. Kluft C. Brommer EJP. The acute effect of smoking on fibrinolysis. Eur J Clin Invest 1984:14: 354-61. 2. Allen RA. Kluft C. Brommer EJP. Effect of chronic smoking on fibrinolysis. Arteriosclerosis
1985:5: 443-50.
D. Influence of cider on the fibrinolytic enzyme system. Acta

on the ESR and the fibrinogen level in healthy subjects. Clin Lab Haematol 1983:5: 45-54.

5. Baker IA Eath R Elewood PC. Etherington M. O'Brien JR. Sweetnam PM. Haemostatic factors associated with ischaemic heart disease in men aged 45-64 years: the Speedwell study. Br Heart J 1982:47: 490-4.

6. Balleisen L. Bailey J. Epping PH. Schulte H, van de Loo J. Epidemiological study on factor VII. factor VIII and fibrinogen in an industrial population. Thromb Haemostas 1985:54: 475-9.

7. Boniton-Kopp C. Scarabin P. Bara L, Castanier M, Jacqueson A. Roger M. Relationship between sex hormones and haemostatic factors in healthy middle-aged men. Atherosclerosis 1988:71: 71-6.

8. Burr ML. Fehily AM. Butland BK. Alcohol and high-density-lipoprotein cholesterol: a randomized controlled trial. Br J Nutr 1986:56: 81-6.

9. Chakrabarti R Overview of fibrinolysis. Scott Med J 1981: S1-S6.

10. Conway $S$. Mauceri $H$. The influence of acute ethanol exposure on growth hormone release in female rats. Alcohol 1991:8: 159-64.

11. Datta R. Sherman ML. Kufe DW. Regulation of proto-oncogene and tumor necrosis factor gene expression by ethanol in HL-60 myeloid leukemia cells. Blood 1990:76: 298-301.

12. Elmer $D$. Gransson' $G$. Zoucas $E$. Impairment of primary hemostasis and platelet function after alcuhol ingestion in man. Haemostasis 1984:14: 223-8.

13. Emeis JJ. Mechanisms involved in short-term changes in blood levels of t-PA. In: Kluft C. ed. Tissue-Iype plasminogen activator (t-PA): physiological and clinical aspects. CRC Press. Boca Ration. FL: 21-35 (1988).

14. Etingin OR. Hajjar DP, Haijar KA. Harpel PC. Nachman RL. Lipoprotein (a) regulates plasminogen activator inhibitor- 1 expression in endothelial cells. A potential mechanism in thrombogenesis. J Biol Chem 1991:266: 2459-65.

15. Fearnley GR. Balmforth G. Fearnley E. Evidence of a diurnal fibrinolytic rhythm: with a simple method of measuring natural fibrinolysis. Clin Sci 1957:16: 645-50.

16. Fearnley GR. Ferguson J. Cliakrabarti R. Vincent CT. Effect of beer on blood fibrinolytic activity. Lancet 1960;i: 184-6.

17. de Geus EJC. Kluft C. de Bart ACW. van Doornen LJP. Effects of exercise training on plasminogen activator inhibitor activity. blood pressure, body fal and the lipid profile. Submilted (1991).

18. Hamsien A. Iselius L. De Faire U. Blombaick M. Genetic and cultural inheritance of plasma fibrinogen concentrations. Lancet 1987:ii: 988-90.

19. Hatake K. Taniguchi T, Ouchi H. Sakaki N. Hishida S. Ijiri I. Possible involvement of kinins in cardiovascular changes after alcohol intake. Pharmacol Biochem Behav 1990:35: 437-42.

20. Haverkate F. Timan G. Preparation of plasminogen-free fibrinogen for fibrin plates. In Davidson JF. Samama MM. Desnoyers PC. eds. Progress in chemical fibrinolysis and thrombolysis. Vol 2. Raven Press. New York: 73-77 (1976).

21. Hillhom M. Kaste M. Rasi V. Effects of ethanol on the hemocoagulation in healthy male volunteers. Acta Neurol Scand 1982:65: 182-3.

22. Hillbom M. Kaste M. Rasi V. Can ethanol intoxication affect hemocoagulation to increase the risk of brain infarction in young adults? Neurology 1983:33: 381-4.

23. Iso $\mathrm{H}$. Folsom $A R$. Wu KK et al. Environmental and genetic variations associated with plasm lso $H$. Folsom AR. Wu KK. et al. Environmental and genetic variations associated with
fibrinogen in Japanese and Caucasians. Thromb Haemostas 1991:65: 816. abstract 469

24. Jackson $R$. Scragg $R$. Beaglehole $R$. Alcohol consumption and risk of coronary heart disease. Bril Med J 1991:303: 211-6.

25. Johansen LG. de Knijff P. Rosseneu H-Y. Havekes LM. Jespersen J. Lipoprotein (a). a rist marker of ischaemic heart disease. High risk profile in Greenland Eskimos - an Inuit population with a reduced risk of acute ischaemic heart disease. Submitted (1991).

26. Kawano S. Masuda E. Tsuji S. Nagano K. Fusamoto H. Kamada T. Ethanol causes vasoconsıriction due to endothelin-1 release in rabbit gastric vessels. Microvasc Res 1991:41: $408-10$ 
27. Kervinen K. Savolainen MJ, Kesaniemi YA. A rapid increase in lipoprotein (a) levels after ethanol withdrawal in alcoholic men. Life Sci 1991:48: 2183-8.

28. Klöcking HP, Hoffmann A. Sonntag G. Acute release of plasminogen activator by ethyl alcohol in the isolated perfused pig ear. Pharmazie 1990:45: 378-9.

29. Kluft C. Jie AFH. Underestimation of tissue-type plasminogen activator activity in plasma when euglobulin fractions are used for assessment. Fibrinolysis 1986;suppl 1, abstract 147.

30. Kluft C. I-PA in fibrin dissolution and hemostasis. In: Kluft C, ed. Tissue-type plasminogen activator (1-PA): physiological and clinical aspects. CRC Press, Boca Raton, FL: $47-79$ (1988)

31. Kluft $C$. Endothelium as a source of tissue-type plasminogen activator ( $t-P A)$ for fibrinolysis. In: Warren JB. ed. The endothelium: An introduction to current research. John Wiley/Alan R. Liss. New York: 129-39 (1990).

32. Kluft C. Disorders of the haemostatic system and the risk of development of thrombotic and cardio-vascular diseases: limitations of laboratory diagnosis. Am J Obstet Gynecol 1990;163: 305-12.

33. Kluft $\mathrm{C}$. Andreotti $\mathrm{F}$. Consequences of the circadian fluctuation in plasminogen activator inhibitor 1 (PAl-1) for studies on blood fibrinolysis. Fibrinolysis 1988:2 suppl 2: 93-5.

34. Kluft C. Verheijen JH. Leiden fibrinolysis working party: Blood collection and handling procedures for assessment of tissue-type plasminogen activator (t-PA) and plasminogen procedures for assessment of tissue-type plasminogen activator (t-PA)
activator inhibitor-1 (PAI-1). Fibrinolysis 1990:4 suppl 2: 155-61.

35. Kluft C. Veenstra J. Schaafsma G. Pikaar NA. Regular moderate wine consumption for five weeks increases plasma activity of the plasminogen activator inhibitor 1 (PAI-1) in healthy young volunteers. Fibrinolysis 1990:11 suppl 2: 69-70.

36. Kooistra T. van den Berg J. Töns A. Platenburg G. Rijken DC. van den Berg E. Butyrate stimulates tissue-type plasminogen-activator synthesis in cultured human endothelial cells. Biochem J 1987;247: 605-12.

37. Lascalzo J. Weinfeld M. Fless GM. Scanu AM. Lipoprotein (a). fibrin binding. and plasminogen activation. Arteriosclerosis 1990:10: 240-5.

38. Laug WE. Ethyl alcohol enhances plasminogen activator secretion by endothelial cells. JAMA 1983:250: $772-6$.

39. de Maat MPM. Kluft C. de Boer K. Knot EAR. Jie AFH. Acid treatment of plasma for the inactivation of plasminogen activator inhibitor-1 (PAI-1). Thromb Res 1988:52: 425 -30.

40. Meade TW. North WRS. Chakrabarti R. Haines AP. Stirling Y. Population-based distributions of haemostatic variables. Br Med Bull 1977:33: 283-8.

41. Meade TW. Chakrabarti R. Haines AP. North WRS. Stirling Y. Characteristics affecting Fibrinolytic activity and plasma fibrinogen concentrations. Br Med J 1979:1: 153-6.

42. Mettinger KL. A study of hemostasis in ischemic cerebrovascular disease. $V$. A multivariate evaluation of risk indicators and predictors. Early results of a longitudinal study. Thromb Res 1982:26: 203-10.

43. Maller $L$. Kristensen TS. Plasma fibrinogen and ischemic heart disease risk factors. Arterioscler Thromb 1991:11: 344-50.

44. Moore RD. Pearson TA. Moderate alcohol consumption and coronary artery disease. Medicine 1986:65: 242-67.

45. Nilsson IM. Bjorkman SE. Van Studnitz W. Hallen A. Antifibrinolytic activity of certain pectins. Thromb Diath Haemorrh 1962:6: 177-87.

46. Ogston $D$. Lea AGH. Langhorn $P$. Wilson SB. The influence of the polyphenols of cider on plasmin and plasminogen activators. Br J Haematol 1985:60: 705-13.

47. Olsen $\mathbf{H}$. Østerud B. Effects of ethanol on human blood fibrinolysis and coagulation. Alcohol Alcoholism 1987. suppl 1: 591-4.

48. Pikaar NA. Wedel M, van der Beek E. van Dokkum W. Kempen HJM, Kluft C, Ockhuizen Th Hermus RJJ. Effects of moderate alcohol consumption on platelet aggregation. fibrinolysis and blood lipids. Metabolism 1987:36: 538-43.

49. Rảnby M. Sundell IB, Nilsson TK. Blood collection in strong acid citrate anticoagulant used in a study of dietary influence on basal t-PA activity. Thromb Haemostas 1989:62: 917-22.

50. Rogers S. Yarnell JWG, Fehily M. Nutritional determinants of haemostatic factors in the Caerphilly study. Eur J Clin Nutr 1988:42: 197-205.
51. Steinberg D, Pearson TA, Kuller LH. Alcohol and atherosclerosis. Ann Intern Med 1991:114: 967-76.

52. Sumi $\mathrm{H}$, Hamada $\mathrm{H}$. Tsushima $\mathrm{H}$, Mihara $\mathrm{H}$. Urokinase-like plasminogen activator increased in plasma after alcohol drinking. Alcohol Alcoholism 1988;23: 33-43.

53. Tran-Thang C. Fasel-Felley J. Pralong G, Hofstetter JR. Bachmann F, Kruithof EKO Plasminogen activators and plasminogen activator inhibitors in liver deficiencies caused by chronic alcoholism or infectious hepatitis. Thromb Haemostas 1989;62: 651-3.

54. Utermann G. The mysteries of lipoprotein (a). Science 1989;246: 904-10.

55. Välimäki M. Härkönen M. Ylikahri R. Acute effects of alcohol on female sex hormones. Alcoholism: Clin Exp Res 1983:7: 289-93.

56. Veenstra J. Moderate alcohol use and coronary heart disease: a u-shaped curve? Wld Rev Nutr Dietet 1991:65: 38-71.

57. Veenstra J. Kluft C. Ockhuizen Th.. van der Pol H, Wedel M. Schaafsma G. Effects of moderate alcohol consumption on platelet function, tissue-type plasminogen activator and plasminogen activator inhibitor. Thromb Haemostas 1990:63: 345-8.

58. Veenstra J. Kluft C. Ockhuizen Th, Pikaar NA. van der Pol H, Wedel M. Schaafsma G. Effects of four days of.moderate wine and coffee consumption on fibrinolysis and platelet aggregation. Fibrinolysis 1990):4: 2(5-220.

59. Veenstra J. Kluft C. van der Pol H. Schaafsma G. Acute effects of moderate alcohol consumption on fibrinolytic factors in healthy middle-aged men. (1991) submitted for publication

60. Verheijen JH. Chang GTG. Kluff C. Evidence of the occurrence of a fast-acting inhibitor of tissue-lype plasminogen activator in human plasma. Thromb Haemostas 1984:51: 392-5.

61. Walter E. Birkholz LB. Harenberg J. Weber E. Circadian rhythms of platelet function, fibrinolytic activity of plasma. and the influence of reduced food uplake. fat loading and beer drinking on fibrinolytic activity. In: Neri Serneri GG. Prentice CRM, eds. Haemostasis and Thrombosis. Academic Press. London: 525-30 (1979).

62. Wejkum L. Rosen S. Sorskog L. Brandt B. Chmielewska J. Blood sampling and determination of tissue plasminogen activator activity with COA-SET t-PA. Fibrinolysis 1990:4 (suppl 2): $152-4$.

63. Yarnell JWG. Fehily AM. Milbank J. Kubicki AJ. Eastham R. Hayes TM. Determinants of plasma lipoproteins and coagulation factors in men from Caerphilly. South Wales. J Epidemiol Community Health 1983:37: 137-40. 